\title{
Piecewise Linear Equalizer for DML based PAM-4 Signal Transmission over a Dispersion Uncompensated Link
}

Fu, Yan; Kong, Deming; Xin, Haiyun; Jia, Shi; Zhang, Kuo; Bi, Meihua; Hu, Weisheng; Hu, Hao

Published in:

Journal of Lightwave Technology

Link to article, DOI:

$10.1109 / J L T .2019 .2948096$

Publication date:

2019

Document Version

Peer reviewed version

Link back to DTU Orbit

Citation (APA):

Fu, Y., Kong, D., Xin, H., Jia, S., Zhang, K., Bi, M., Hu, W., \& Hu, H. (2019). Piecewise Linear Equalizer for DML based PAM-4 Signal Transmission over a Dispersion Uncompensated Link. Journal of Lightwave Technology, 38(3), 654 - 660. https://doi.org/10.1109/JLT.2019.2948096

\section{General rights}

Copyright and moral rights for the publications made accessible in the public portal are retained by the authors and/or other copyright owners and it is a condition of accessing publications that users recognise and abide by the legal requirements associated with these rights.

- Users may download and print one copy of any publication from the public portal for the purpose of private study or research.

- You may not further distribute the material or use it for any profit-making activity or commercial gain

- You may freely distribute the URL identifying the publication in the public portal 


\title{
Piecewise Linear Equalizer for DML based PAM-4 Signal Transmission over a Dispersion Uncompensated Link
}

\author{
Yan Fu, Deming Kong, Haiyun Xin, Shi Jia, Kuo Zhang, Meihua Bi, Weisheng Hu, and Hao Hu
}

\begin{abstract}
Directly modulated laser (DML) and direct detection (DD) based pulse-amplitude modulation (PAM) for short reach optical communications has attracted lots of research interests due to its low cost and simple configuration. However, the directly modulated PAM signal usually suffers from distortions after the transmission over a dispersion uncompensated link. Due to the adiabatic chirp of the DML working in a high output power region, different intensity levels of the PAM signal correspond to different frequency offsets, thus resulting in amplitude-dependent skew after the transmission over a dispersion uncompensated link. It will degrade signal quality and limit transmission distance. In this paper, we, for the first time, propose a computational efficient piecewise linear (PWL) equalizer to correct the amplitude-dependent skew due to the interaction between the DML chirp and chromatic dispersion in the fiber. By using the PWL equalizer, the amplitude-dependent skew is corrected, and we successfully transmit 56 Gb/s PAM-4 signal over $40 \mathrm{~km}$ and 84 Gb/s PAM-4 signal over $20 \mathrm{~km}$ dispersion uncompensated link in the $\mathrm{C}$ band, with a bit error ratio (BER) below the HD-FEC threshold $\left(3.8 \times 10^{-3}\right)$. To demonstrate the low computational complexity of the PWL, we compare its complexity with $2^{\text {nd }}$ order Volterra equalizer for a similar BER performance. The results show that the computational complexity can be reduced by $61.4 \%$ and $40.4 \%$ for the $56 \mathrm{~Gb} / \mathrm{s}$ and $84 \mathrm{~Gb} / \mathrm{s}$ PAM-4 signal transmission, respectively.
\end{abstract}

Index Terms-Linear equalizer, direct-modulated laser, pulse-amplitude modulation, direct detection, digital signal processing.

This work was partially supported by the National Natural Science Foundation of China under Grant 61431009, Villum Young Investigator program (2MAC), and China Scholarship Council (CSC) under grant 201806230097 (Corresponding author: Hao Hu).

$\mathrm{Y}$. $\mathrm{Fu}$, and $\mathrm{H}$. Xin are with the Shanghai Institute for Advanced Communication and Data Science, State Key Laboratory of Advanced Optical Communication Systems and Networks, Shanghai Jiao Tong University, Shanghai 200240, China, also with the DTU Fotonik, Technical University of Denmark, Kongens Lyngby 2800, Denmark (email: fu_yan@sjtu.edu.cn; haixin@ fotonik.dtu.dk).

D. Kong, S. Jia, and H. Hu are with the DTU Fotonik, Technical University of Denmark, Kongens Lyngby 2800, Denmark (email: dmkon@fotonik.dtu.dk; shijai@fotonik.dtu.dk; huhao@ fotonik.dtu.dk )

K. Zhang and W. Hu are with the Shanghai Institute for Advanced Communication and Data Science, State Key Laboratory of Advanced Optical Communication Systems and Networks, Shanghai Jiao Tong University, Shanghai 200240, China (email: zhangkuo91@126.com; wshu@ sjtu.edu.cn)

$\mathrm{M}$. Bi is with the School of Communication Engineering, Hangzhou Dianzi University, Hangzhou, Zhejiang 310018, China (email: bmhua@ hdu.edu.cn).

\section{INTRODUCTION}

C PURRED by the rapid growth of high-bandwidth internet $\checkmark$ applications such as cloud services, streamed video, and Internet of Things, the bandwidth requirement of intra and inter-data center interconnection (DCI) is continuously increasing. For the DCI applications, the intensity modulation and direct detection (IM/DD) systems have attracted many research interests due to their low cost, small footprint, and simple configuration [1], [2]. Several advanced modulation formats have been proposed for the IM/DD system, such as pulse-amplitude-modulation with four amplitude levels (PAM-4), discrete multi-tone modulation (DMT), and carrier-less amplitude-phase modulation (CAP) [3], [4]. PAM-4 has the advantage of only requiring 2-bit digital-to-analog converters (DAC), thus simplifying the transmitter. In the IM/DD systems, directly modulated laser (DML) based transmitter is more desirable than externally modulated laser (EML), and Mach-Zehnder modulator (MZM) based transmitters due to lower cost and lower power consumption. However, DML suffers from frequency chirp, consisting of adiabatic chirp and transient chirp. When a DML is biased at high current, the adiabatic chirp is dominant [5], [6]. In that case, different intensity levels of PAM signal correspond to different frequency offsets and accordingly transmit at different velocities through a dispersive fiber. As a consequence, the eye diagram of the PAM signal after the transmission exhibits an eye skewing effect, which limits both the extinction ratio (ER) and transmission distance [7]. Several techniques have been proposed to solve this problem. The first is transmitting signal using DMLs in O band [8], [9] because chromatic dispersion is minimum. Nevertheless, $\mathrm{O}$ band transmission suffers from high fiber attenuation. The second is utilizing vestigial sideband (VSB) modulation since filtering out one sideband can eliminate power fading caused by chromatic dispersion [10]. However, VSB modulation has stringent requirements on the bandwidth and central wavelength of the optical filter. The third solution is using either Volterra series nonlinear equalizers or neural network algorithm based equalizers [11]-[14]. However, the nonlinear equalizer usually suffers from high computational complexity, and the neural network algorithm relies on a large amount of training data and computing resources. Alternatively, this eye skewing problem can be solved with two steps, skew estimation 
and skew compensation, to reduce the complexity [15], [16]. In [15], the proposed nonlinear skew compensator is designed to apply amplitude-dependent delay on incoming pulses to mitigate the eye skew of the DML-based PAM signal. In [16], the skew estimation is achieved by the minimum standard deviation of each level over one unit interval. In addition, the intensity-directed equalizer was also used to solve this problem by classifying a symbol into different segments according to its intensity level [17], [18]. However, these solutions are intrinsically based on classification function, which is not reliable to model the severe nonlinear response, otherwise much more computational complexity is required to correct eye skew.

Based on the concept that nonlinear input/output transmission response can be approximated as low-dimensional expansions, the low-dimensional elements, such as piecewise linear approximations, were successfully applied for acoustic echo cancellation where a power amplifier and a loudspeaker induce a nonlinear echo that degrades the output signal [19], [20]. Inspired by that, to the best of our knowledge, it is the first time the piecewise linear equalizer is applied in an IM/DD system to correct eye skew by using the amplitude threshold decomposition. $56 \mathrm{~Gb} / \mathrm{s}$ and $84 \mathrm{~Gb} / \mathrm{s}$ [21], [22] PAM-4 signal using DML at high output power region with ERs of $5.2 \mathrm{~dB}$ and $4.2 \mathrm{~dB}$ are transmitted over $40 \mathrm{~km}$ and $20 \mathrm{~km}$ dispersion unmanaged links below the hard-decision forward error correction (HD-FEC) threshold, while achieving a similar BER performance as a $2^{\text {nd }}$ order Volterra equalizer yet with $61.4 \%$ and $40.4 \%$ computational complexity reduction.

The paper is organized as follows: The principle of PWL equalizer is explained in Section II. The experimental setup is described in Section III. The experimental results are discussed in Section IV, including amplitude threshold sets optimization, transmission performances using the PWL, performance of the PWL equalizer for various DML driving voltages, and computational complexity comparison between the PWL and $2^{\text {nd }}$ order Volterra equalizer. The results are summarized and concluded in Section V.

\section{PRINCIPLE OF PIECEWISE LINEAR EQUALIZER}

As presented in [20], the PWL equalizer has been successfully applied in acoustic echo cancellation, and it is proved that the PWL performs well for memoryless nonlinearities. Due to this advantage, the PWL equalizer can be used in DML-DD systems for compensating the nonlinear distortions introduced by the DML chirp and fiber chromatic dispersion. The PWL equalizer consists of three steps (see Fig. 1), including amplitude threshold decomposition, linear multichannel equalization, and linear addition. In the first step, by using $N$ pre-defined thresholds $\tau=\left\{\lambda_{1}, \lambda_{2} \ldots, \lambda_{N-1}, \lambda_{N}\right\}$, the real space can be partitioned into $N+1$ disjoint intervals: $I_{1}=\left(-\infty, \lambda_{1}\right) \quad, \quad I_{2}=\left[\lambda_{1}, \lambda_{2}\right) \quad \ldots, \quad I_{N}=\left[\lambda_{N-1}, \lambda_{N}\right) \quad, \quad$ and $I_{N+1}=\left[\lambda_{N}, \infty\right)$. When intervals are given, the corresponding decomposed segments: $M_{1}, M_{2} \ldots, M_{N-1}, M_{N}$, and $M_{N+1}$ can be acquired. The rules to get $i$ th segment $M_{i},(i=1,2, \ldots, N+1)$ is formulated in Table $\mathrm{I}$.

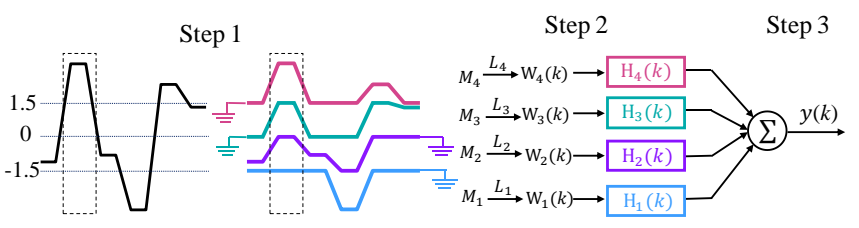

Fig. 1. Piecewise linear equalizer procedures, step 1: threshold decomposition with the threshold set $\{-1.5,0,1.5\}$ for the signal sequence

$[-1.1,3.2,-0.8,-3.2,2.3,1.3]$, step 2 : linear equalization and step 3: addition.

TABLE I THRESHOLD DECOMPOSITION ALGORITHM: THE RULES TO GET

\begin{tabular}{lllll}
\multicolumn{5}{c}{$M_{i},(i=1,2, \ldots, N+1)$} \\
\hline \hline \multirow{2}{*}{$\gamma \geq 0$} & $\lambda_{i-1}>0$ & $\lambda_{i-1} \leq 0 \leq \lambda_{i}$ & $\lambda_{i}<0$ \\
& $\gamma \in I_{i}$ & $\gamma-\lambda_{i-1}$ & $\gamma$ & $\varnothing$ \\
& $\gamma>\lambda_{i}$ & $\lambda_{i}-\lambda_{i-1}$ & $\lambda_{i}$ & 0 \\
& $\gamma \leq \lambda_{i-1}$ & 0 & $\varnothing$ & $\varnothing$ \\
\hline \multirow{2}{*}{$\gamma<0$} & $\gamma \in I_{i}$ & $\varnothing$ & $\gamma$ & $\gamma-\lambda_{i}$ \\
& $\gamma>\lambda_{i}$ & $\varnothing$ & $\varnothing$ & 0 \\
& $\gamma \leq \lambda_{i-1}$ & 0 & $\lambda_{i-1}$ & $\lambda_{i-1}-\lambda_{i}$ \\
\hline \hline
\end{tabular}

The threshold decomposition process of the PAM-4 samples $\mathbf{X}=[-1.1,3.2,-0.8,-3.2,2.3,1.3]$ is shown in Fig. 1, where the threshold set: $\tau=\left\{\lambda_{1}, \lambda_{2}, \lambda_{3}\right\}=\{-1.5,0,1.5\}$ is used to split $\mathbf{X}$ into four subintervals: $I_{1}=(-\infty,-1.5), I_{2}=[-1.5,0)$, $I_{3}=[0,1.5)$, and $I_{4}=[1.5, \infty)$. We then take the second sample, i.e., $\gamma=\mathbf{X}(2)=3.2$, to explain the rules in Table I. Since $\gamma \geq 0, \quad \gamma \in I_{4}$, and $\lambda_{3}>0$, we can get $M_{4}(2)=\gamma-\lambda_{3}=3.2-1.5=1.7$. Similarly, $\quad M_{3}(2)=1.5 \quad$, $M_{2}(2)=0$, and $M_{1}(2)=0$ can be obtained.

The second step of PWL equalizer consists of $N+1$ linear equalizers (e.g., feed-forward equalizer (FFE)) in parallel for $N+1$ decomposed segments. Given the present sample for $M_{i}$ is $M_{i}(k), \mathbf{W}_{i}(k)$ is given in (1) $i=1,2, \ldots, N+1$. The equalizer weighting factors for $\mathbf{W}_{i}(k)$ is given by $\mathbf{H}_{i}(k)$ with the number of $L_{i}$ taps,

$$
\mathbf{W}_{i}(k)=\left[\begin{array}{l}
M_{i}(k) \\
M_{i}(k-1) \\
\cdots \\
M_{i}\left(k-L_{i}+1\right)
\end{array}\right], \mathbf{H}_{i}(k)=\left[\begin{array}{l}
h_{i 1}(k) \\
h_{i 2}(k) \\
\ldots \\
h_{i L_{i}}(k)
\end{array}\right]
$$

The output values of $N+1$ parallel equalizers are summed in the third step. The summed output value of the finite impulse response (FIR) filter $y(k)$ is a product between the input $\mathbf{W}_{i}(k)$ and a transposed vector of $\mathbf{H}_{i}(k)$,

$$
y(k)=\sum_{i=1}^{N+1} \mathbf{H}_{i}{ }^{T}(k) \mathbf{W}_{i}(k) .
$$


When we know the output of the process, the error $\varepsilon(k)$ is expressed as the difference between the transmitted signal sample $d(k)$ and output $y(k)$,

$$
\varepsilon(k)=d(k)-y(k) .
$$

Hereafter, the weightings of the filter are updated to minimize the output mean squared error $E\left[(d(k)-y(k))^{2}\right]$. Then, the weighting vector $\mathbf{H}_{i}(k+1)$ for the next iteration can be got from the sum of the current weighting vector $\mathbf{H}_{i}(k)$ with the weighted input vector $\mathbf{W}_{i}(k)$, and the input vector is scaled with the error value $\varepsilon(k)$ and the adaptation rate $\mu_{i}$,

$$
\mathbf{H}_{i}(k+1)=\mathbf{H}_{i}(k)+\mu_{i} \varepsilon(k) \mathbf{W}_{i}(k) \text {. }
$$
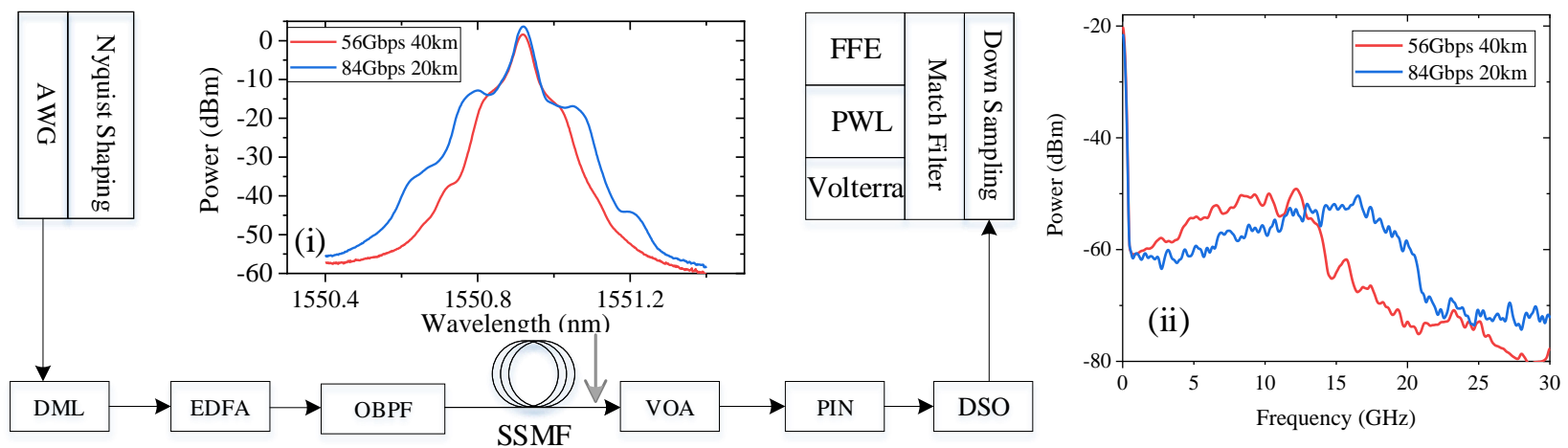

Fig. 2. Experimental setup of $56 \mathrm{~Gb} / \mathrm{s}$ and $84 \mathrm{~Gb} / \mathrm{s}$ PAM-4 signals transmission based on the DML-DD system. (i) and (ii): Optical spectra and electrical power spectra of $56 \mathrm{~Gb} / \mathrm{s}$ PAM- 4 over $40 \mathrm{~km}$ and $84 \mathrm{~Gb} / \mathrm{s}$ PAM- 4 over $20 \mathrm{~km}$ transmissions.

\section{EXPERIMENTAL SETUP}

As shown in Fig. 2, $56 \mathrm{~Gb} / \mathrm{s}$ and $84 \mathrm{~Gb} / \mathrm{s}$ baseband electrical PAM-4 signals with a $2^{15}-1$ bits and a $2^{11}-1$ bits pseudo-random bit sequence (PRBS) using Nyquist pulse shaping generator (AWG) with a roll-off factor of 0.01 are generated by an arbitrary waveform generator (AWG) (Keysight M8195A). The AWG has a sampling rate of 65 $\mathrm{GSa} / \mathrm{s}$, a bandwidth of $25 \mathrm{GHz}$, a resolution of 8 bits, and up to $16 \mathrm{GSa}$ of waveform memory. When working with $84 \mathrm{~Gb} / \mathrm{s}$ PAM-4 signal, $2^{15}-1$ bits PRBS can't be selected on the AWG in the experiment due to practical limitations. Therefore, we use $2^{11}-1$ bits PRBS for $84 \mathrm{~Gb} / \mathrm{s}$ PAM-4. The peak-to-peak voltage (Vpp) of electrical PAM-4 signal in AWG is adjusted to $0.35 \mathrm{~V}, 0.45 \mathrm{~V}, 0.55 \mathrm{~V}, 0.65 \mathrm{~V}, 0.75 \mathrm{~V}, 0.85 \mathrm{~V}$ and $0.95 \mathrm{~V}$ for both $56 \mathrm{~Gb} / \mathrm{s}$ and $84 \mathrm{~Gb} / \mathrm{s}$ PAM-4 signal, then a 6-dB RF attenuator is placed before the $26 \mathrm{~dB}$ RF amplifier (SHF806A) to enable the RF amplifier to work in linear region. After that, one 6-dB RF attenuator is placed after the RF amplifier to reduce the amplitude. Therefore, the corresponding Vpps of electrical signal driving DML are $1.75 \mathrm{~V}, 2.25 \mathrm{~V}, 2.75 \mathrm{~V}, 3.25$ $\mathrm{V}, 3.75 \mathrm{~V}, 4.25 \mathrm{~V}$ and 4.75 V. The DML used in the experiment has a center wavelength of $1550.9 \mathrm{~nm}$ and a $3 \mathrm{~dB}$ bandwidth of $\sim 20 \mathrm{GHz}$, which is biased at $98 \mathrm{~mA}$ and modulated by the electrical signals to produce a PAM-4 signal with an output
The computational complexity of the PWL equalizer can be expressed as:

$$
C C_{P W L}=\sum_{i=1}^{N+1} L_{i} \cdot
$$

From this equation, we know that the complexity of PWL equalizer is $N+1$ times of that of standard FFE. We will demonstrate that PWL equalizer with threshold sets of $\tau=\{-\lambda, 0, \lambda\}$ and $\tau=\{-\lambda, \lambda\}$ can meet an acceptable BER performance in equalizing PAM-4 signals using DML at a high output power region with high ERs in the following sections. Thus, the computational complexity of the PWL equalizer is four or three times of that of conventional FFE. power of $7.4 \mathrm{dBm}$. The corresponding ERs are $2.8 \mathrm{~dB}, 3.6 \mathrm{~dB}$, $4.4 \mathrm{~dB}, 5.2 \mathrm{~dB}, 5.6 \mathrm{~dB}, 6.2 \mathrm{~dB}, 6.9 \mathrm{~dB}$ for the $56 \mathrm{~Gb} / \mathrm{s}$ PAM-4 signal and $2.4 \mathrm{~dB}, 3.1 \mathrm{~dB}, 3.6 \mathrm{~dB}, 4.2 \mathrm{~dB}, 4.5 \mathrm{~dB}, 5.4 \mathrm{~dB}$, $5.7 \mathrm{~dB}$ for the $84 \mathrm{~Gb} / \mathrm{s}$ PAM-4 signal. When the DML is operating at a high bias current, the adiabatic chirp induced adiabatic fading can effectively compensate the first transient fading notch, thereby adiabatic fading is dominant [5]. The PWL equalizer is quite effective for the adiabatic chirp dominant case. An optical bandpass filter (OBPF) with a bandwidth of $1 \mathrm{~nm}$ is used after an erbium-doped fiber amplifier (EDFA) to remove the out-of-band noise. The launched powers into $20 \mathrm{~km}$ and $40 \mathrm{~km} \mathrm{SSMFs} \mathrm{for} 56 \mathrm{~Gb} / \mathrm{s}$ signal, and into $20 \mathrm{~km} \mathrm{SSMF}$ for $84 \mathrm{~Gb} / \mathrm{s}$ signal, are $16 \mathrm{dBm}$ and $14 \mathrm{dBm}$, respectively. The optical spectra of $56 \mathrm{~Gb} / \mathrm{s}$ PAM-4 over $40 \mathrm{~km}$ and $84 \mathrm{~Gb} / \mathrm{s}$ PAM-4 over $20 \mathrm{~km}$ transmissions are shown in Fig. 2(i). A variable optical attenuator (VOA) is used to adjust the received optical power (ROP) into the photodiode (PD) with $70 \mathrm{GHz}$ bandwidth. There is no integrated transimpedance amplifier (TIA) or electrical amplifier used after the PD. The received signal is sampled by a digital storage oscilloscope (DSO) with a sampling rate of 80 $\mathrm{GSa} / \mathrm{s}$ and $32 \mathrm{GHz}$ bandwidth. For the digital signal processing in the receiver, the signal samples are sent into the match filter with a roll-off factor of 0.01 , then down sampled to two samples per symbol and equalized by the proposed PWL 
equalizer. The BER performance is compared with conventional FFE and Volterra equalizers. A total number of 32768 symbols are used to evaluate the BER. In the experiment, we choose threshold sets with three or two thresholds to test the performance of the PWL equalizer: $\tau=\{-\lambda, 0, \lambda\}$ and $\tau=\{-\lambda, \lambda\}$. The electrical power spectra of $56 \mathrm{~Gb} / \mathrm{s}$ and 84 Gb/s PAM-4 signal are shown in Fig. 2(ii). We can observe that the first power fading notch points locate in $14.67 \mathrm{GHz}$ and 22.67 GHz for the $56 \mathrm{~Gb} / \mathrm{s}$ and $84 \mathrm{~Gb} / \mathrm{s}$ PAM-4 signal transmission over $40 \mathrm{~km}$ and $20 \mathrm{~km} \mathrm{SSMF}$, respectively.

\section{EXPERIMENTAL RESULTS}

\section{A. Threshold Setting Optimization}

Two threshold sets $\{-\lambda, 0, \lambda\}$ and $\{-\lambda, \lambda\}$ are tested for the PWL equalizer, which means four or three parallel FFEs are used. A conventional 11-tap FFE (pre-equalizer) is used before the parallel 15-tap FFEs $(56 \mathrm{~Gb} / \mathrm{s})$ or 51-tap FFEs $(84 \mathrm{~Gb} / \mathrm{s})$ for PWL equalizer in the experiment to compensate part of the linear distortions due to chromatic dispersion, and to distribute signal around $[-3,-1,1,3]$ to facilitate accurate segmentation. around $[-3,-1,1,3]$, and most of the pre-equalized signals are within the range of $[-4,4], \lambda$ is varied from 0 to 4 to find the optimum $\lambda$ value for $56 \mathrm{~Gb} / \mathrm{s}$ over $40 \mathrm{~km}$ and $84 \mathrm{~Gb} / \mathrm{s}$ over 20 $\mathrm{km}$ transmission with a fixed ROP of $5.6 \mathrm{dBm}$, as shown in Fig. 3.

For $56 \mathrm{~Gb} / \mathrm{s}$ PAM-4 signal over $40 \mathrm{~km}$ transmission, when the threshold set of $\{-4,4\}$ is used in PWL equalizer, it is the same as a conventional FFE since there is no decomposition. Its eye diagram has a severe skew (see Fig. 3(i)). BER at this threshold set is $2.58 \times 10^{-2}$. The threshold sets of $\{-0,0,0\}$ and $\{-0,0\}$ split the PAM-4 signal space into positive and negative segments. The eye diagram exhibits a significant improvement for the eye skew (see Fig. 3(ii)), and the BER is considerably reduced to $5.06 \times 10^{-3}$. The best BER performances are achieved at the threshold sets of $\{-1,1\}$ and $\{-1.4,0,1.4\}$ for threshold sets $\{-\lambda, \lambda\}$ and $\{-\lambda, 0, \lambda\}$ with BERs of $3.5 \times 10^{-3}$ and $3.6 \times 10^{-3}$. The eye diagram of the former is shown in Fig. 3(iii).

Since the pre-equalizer enables received data to distribute
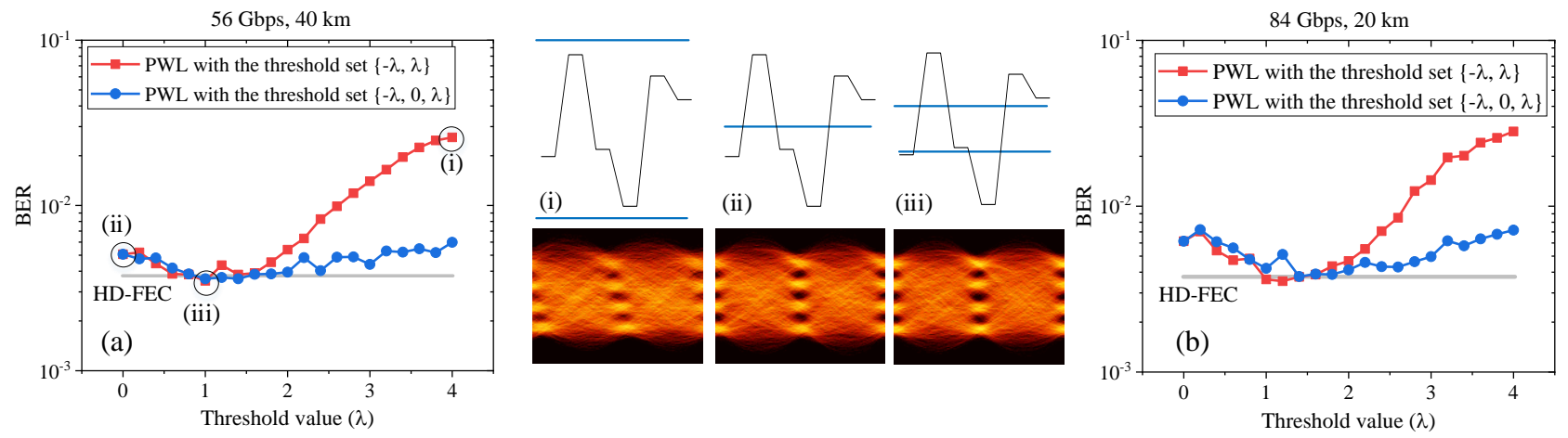

Fig. 3. The relationship between BER performances and threshold $\lambda$ values for two threshold sets: $\{-\lambda, \lambda\}$ and $\{-\lambda, 0, \lambda\}$ of (a) $56 \mathrm{~Gb} / \mathrm{s}$ over $40 \mathrm{~km}$ and (b) 84 $\mathrm{Gb} / \mathrm{s}$ over $20 \mathrm{~km}$ transmissions (b). (i), (ii)and (iii) correspond to the eye diagrams and threshold decompositions schematic diagrams in $56 \mathrm{~Gb} / \mathrm{s}$ over $40 \mathrm{~km}$ transmission for PWL equalizer for threshold sets: $\{-4,4\}\{0\}$, and $\{-1,1\}$, respectively.

TABLE II PARAMETERS OF $56 \mathrm{~GB} / \mathrm{S}$ AND $84 \mathrm{~GB} / \mathrm{S}$ PAM-4 TRANSMISSION.

\begin{tabular}{|c|c|c|c|c|c|}
\hline Bit rate & Distances & $\begin{array}{c}\text { Optimum RF input } \\
\text { Vpps (V) }\end{array}$ & FFE1 & FFE2 & PWL \\
\hline \multirow{3}{*}{$56 \mathrm{~Gb} / \mathrm{s}$} & $\mathrm{BtB}$ & $4.75 \mathrm{~V}$ & & & \\
\hline & $20 \mathrm{~km}$ & $3.75 \mathrm{~V}$ & $\begin{array}{l}\text { 11-tap pre-FFE + } \\
\text { 15-tap FFE }\end{array}$ & $\begin{array}{l}\text { 11-tap pre-FFE + } \\
\text { 45-tap FFE }\end{array}$ & $\begin{array}{l}\text { 11-tap pre-FFE + } 3 \\
\text { parallel } 15 \text {-tap FFEs }\end{array}$ \\
\hline & $40 \mathrm{~km}$ & $3.25 \mathrm{~V}$ & & & \\
\hline \multirow[b]{2}{*}{$84 \mathrm{~Gb} / \mathrm{s}$} & $\mathrm{BtB}$ & $4.75 \mathrm{~V}$ & & & \\
\hline & $20 \mathrm{~km}$ & $3.25 \mathrm{~V}$ & $\begin{array}{l}\text { 11-tap pre-FFE + } \\
\text { 51-tap FFE }\end{array}$ & $\begin{array}{l}\text { 11-tap pre-FFE + } \\
\text { 153-tap FFE }\end{array}$ & $\begin{array}{l}\text { 11-tap pre-FFE }+3 \\
\text { parallel 51-tap FFEs }\end{array}$ \\
\hline
\end{tabular}

Similar BER performance is observed for the case of $84 \mathrm{~Gb} / \mathrm{s}$ PAM-4 over $20 \mathrm{~km}$ transmission using the PWL. For the threshold set of $\{-4,4\}$, the BER is $2.58 \times 10^{-2}$. It decreases to $6.15 \times 10^{-3}$ when the threshold sets $\{-0,0,0\}$ and $\{-0,0\}$ are used. The lowest BERs are achieved for the threshold sets of
$\{-1.2,1.2\}$ and $\{-1.4,0,1.4\}$ with the BERs of $3.53 \times 10^{-3}$ and $3.76 \times 10^{-3}$. When $\lambda$ is larger than 2 , the BER with the threshold set of $\{-\lambda, 0, \lambda\}$ is better than using the threshold set of $\{-\lambda, \lambda\}$ because amplitude threshold decomposition is too unevenly distributed after using the threshold set of $\{-\lambda, \lambda\}$. 
Thanks to the PWL equalizer, $56 \mathrm{~Gb} / \mathrm{s}$ and $84 \mathrm{~Gb} / \mathrm{s}$ PAM-4 signals can be transmitted over $40 \mathrm{~km}$ and $20 \mathrm{~km}$ dispersion uncompensated link with the BERs below the HD-FEC threshold of $3.8 \times 10^{-3}$ with a $7 \%$ FEC overhead, respectively. Since the best BER performances for both threshold sets are similar, we select the threshold sets of $\{-1,1\}$ and $\{-1.2,1.2\}$ for $56 \mathrm{~Gb} / \mathrm{s}$ and $84 \mathrm{~Gb} / \mathrm{s}$ PAM-4 transmission for the rest of the analysis due to lower computational complexity.

\section{B. Transmission Performance over Different Distances}

BER performances as a function of ROP for different conditions are shown in Fig. 4, including the comparisons of PWL equalizer and conventional FFE equalizers. As shown in Table II, the PWL equalizer for $56 \mathrm{~Gb} / \mathrm{s}$ signal consists of 11-tap pre-equalizer and three parallel 15-tap FFEs, totally 56 taps. First, we introduce FFE1 (11-tap pre-equalizer and 15-tap FFE) to analyse if similar BER can be achieved for the back-to-back $(\mathrm{BtB})$ case because three segments of PWL should converge to same equalizer coefficients in linear distortion scenarios. Then, we introduce FFE2 (11-tap pre-equalizer and 45-tap FFE) for the comparison considering its same total equalizer taps with the PWL. The relevant parameters of FFE1, FFE2, and PWL for $84 \mathrm{~Gb} / \mathrm{s}$ PAM-4 are also described in Table II. Besides, the optimal RF input Vpp values for $56 \mathrm{~Gb} / \mathrm{s}$ over $\mathrm{BtB}, 20 \mathrm{~km}$, and $40 \mathrm{~km}$ are $4.75 \mathrm{~V}, 3.75$ $\mathrm{V}$, and $3.25 \mathrm{~V}$, and for $84 \mathrm{~Gb} / \mathrm{s}$ over BtB and $20 \mathrm{~km}$ are $4.75 \mathrm{~V}$ and $3.25 \mathrm{~V}$, respectively.

In the BtB case (Figs. 4(a) and 4(d)), PWL and FFE1 exhibit nearly identical BER performance for both $56 \mathrm{~Gb} / \mathrm{s}$ and $84 \mathrm{~Gb} / \mathrm{s}$ PAM-4, and FFE2 performs slightly better. It is because three parallel FFEs in PWL converge to same equalizer tap coefficients in the absence of nonlinear response, and the dominant linear distortion is unable to be completely compensated by the FFE equalizer with a small number of taps.

In the case of $56 \mathrm{~Gb} / \mathrm{s}$ PAM-4 over $20 \mathrm{~km}$ transmission (Fig. 4(b)), the interaction between the DML adiabatic chirp and fiber chromatic dispersion is gradually dominant compared to linear distortions. Therefore, FFE1 can just reach the HD-FEC limit @ ROP >3.6 dBm, and PWL performs better than FFE2. In this case, the BERs of FFE1, FFE2, and PWL are $3 \times 10^{-3}$, $1.2 \times 10^{-3}$, and $3 \times 10^{-4}$ at the ROP of $5.6 \mathrm{dBm}$, respectively.

In the case of $56 \mathrm{~Gb} / \mathrm{s}$ PAM-4 over $40 \mathrm{~km}$ (Fig. 4(c)) and $84 \mathrm{~Gb} / \mathrm{s}$ PAM-4 over $20 \mathrm{~km}$ (Fig. 4(e)), the signal degradation is mainly attributed to the interaction between the adiabatic chirp and the fiber chromatic dispersion. Also, PWL equalizer is exactly suitable for this scenario. Therefore, the PWL can significantly outperform both the FFE1 and FFE2. Thanks to the PWL equalizer, clear eye openings are observed (Figs. 4(ii) and 4(iv)), compared with the skewed eye diagrams after the FFE2 (Figs. 4(i) and 4(iii)). The BERs of the PAM-4 signal using the PWL at the ROP of $5.6 \mathrm{dBm}$ are $3.6 \times 10^{-3}$ and $3.53 \times 10^{-3}$ for $56 \mathrm{~Gb} / \mathrm{s}$ over $40 \mathrm{~km}$ and $84 \mathrm{~Gb} / \mathrm{s}$ over $20 \mathrm{~km}$ transmissions, respectively. In contrast, the BERs are $1.27 \times 10^{-2}$ and $2.16 \times 10^{-2}$ by using the FFE2. As mentioned above, with EDFA as a booster, HD-FEC limit with $7 \%$ overhead $\left(3.8 \times 10^{-3}\right)$ can be achieved for both $56 \mathrm{~Gb} / \mathrm{s}$ PAM-4 over $40 \mathrm{~km}$ and $84 \mathrm{~Gb} / \mathrm{s}$ PAM-4 over $20 \mathrm{~km}$ transmissions. Without EDFA as a booster, $56 \mathrm{~Gb} / \mathrm{s}$ over $20 \mathrm{~km}$ can still be achieved below HD-FEC with $7 \%$ overhead, but $56 \mathrm{~Gb} / \mathrm{s}$ over $40 \mathrm{~km}$ and $84 \mathrm{~Gb} / \mathrm{s}$ over $20 \mathrm{~km}$ can only support HD-FEC with a higher overhead [23].
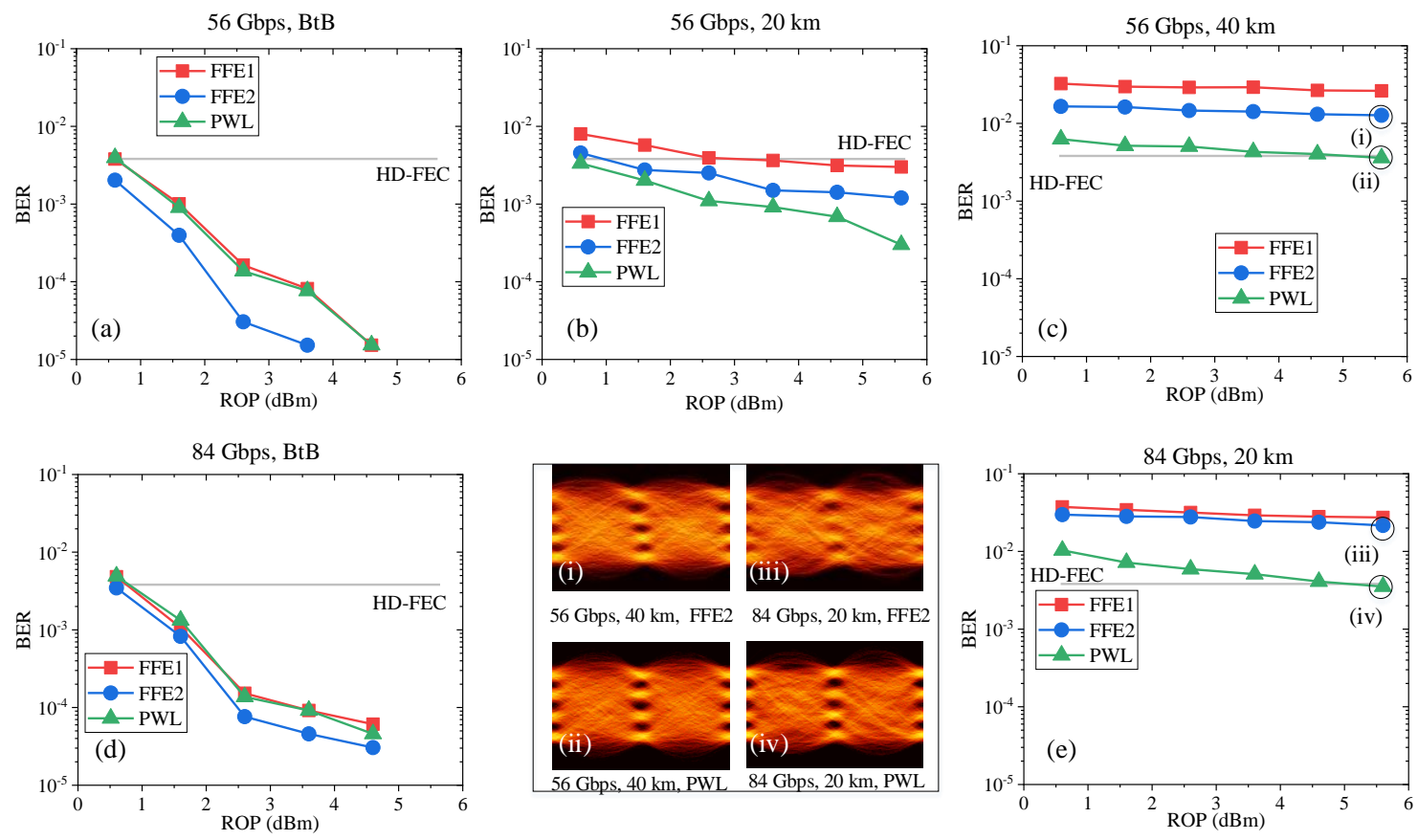

Fig. 4. BER curves versus ROP of $56 \mathrm{~Gb} / \mathrm{s}$ PAM- 4 over BtB, $20 \mathrm{~km}$, and $40 \mathrm{~km}$, and $84 \mathrm{~Gb} / \mathrm{s}$ PAM-4 over BtB, 20 km with FFE1, FFE2, and PWL equalizers. (i) and (ii): Eye diagrams of $56 \mathrm{~Gb} / \mathrm{s}$ over $40 \mathrm{~km}$ case with FFE2 and PWL, (iii) and (iv): Eye diagrams of 84Gb/s over $20 \mathrm{~km}$ case with FFE2 and PWL. 


\section{Performance of the PWL Equalizer for Various DML Driving Voltages}

Fig. 5 compares the BER performances and eye diagrams by using the FFE2 and the PWL when varying PAM-4 RF input Vpps for $56 \mathrm{~Gb} / \mathrm{s}$ over $40 \mathrm{~km}$ and $84 \mathrm{~Gb} / \mathrm{s}$ over $20 \mathrm{~km}$ transmissions with a fixed ROP of $5.6 \mathrm{dBm}$. The RF input Vpps are adjusted to $1.75 \mathrm{~V}, 2.25 \mathrm{~V}, 2.75 \mathrm{~V}, 3.25 \mathrm{~V}, 3.75 \mathrm{~V}, 4.25 \mathrm{~V}$, and $4.75 \mathrm{~V}$, which correspond to ER values of $2.8 \mathrm{~dB}, 3.6 \mathrm{~dB}$, $4.4 \mathrm{~dB}, 5.2 \mathrm{~dB}, 5.6 \mathrm{~dB}, 6.2 \mathrm{~dB}, 6.9 \mathrm{~dB}$ for $56 \mathrm{~Gb} / \mathrm{s}$ PAM- 4 signal and ER values of $2.4 \mathrm{~dB}, 3.1 \mathrm{~dB}, 3.6 \mathrm{~dB}, 4.2 \mathrm{~dB}, 4.5 \mathrm{~dB}$, $5.4 \mathrm{~dB}, 5.7 \mathrm{~dB}$ for $84 \mathrm{~Gb} / \mathrm{s}$ PAM-4 signal.

For the case of low Vpp, the BER performance is limited by the low signal to noise ratio (SNR). While for the case of high Vpp, the BER performance is limited by the severe adiabatic chirp. Both the low SNR and the severe adiabatic chirp can cause closed eyes after the transmission. Only the severe adiabatic chirp leads to the skewed eyes. Therefore, the eye diagrams are more and more skewed with the increase of the

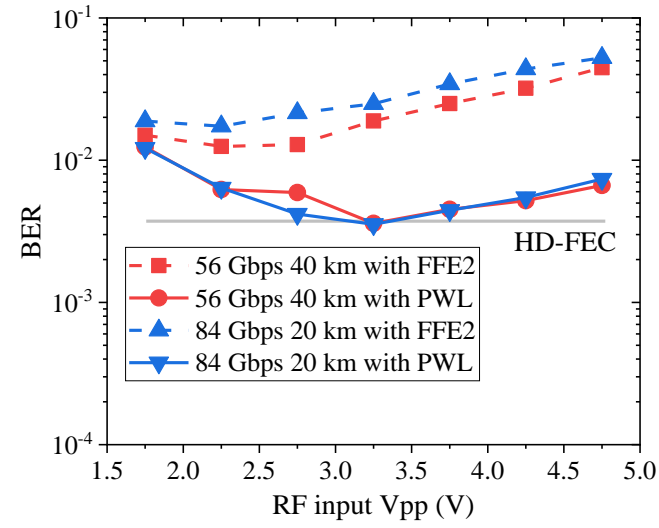

RF Vpp (see Figs. 5(i)-(iii)). Thanks to the PWL equalizer, it can effectively correct the skewed eye diagram in the whole Vpp range, even for the severely skewed eye diagram at the Vpp of $4.75 \mathrm{~V}$, as shown in Figs. 5(iv)-(vi).

However, it is not very helpful for the low SNR induced closed eye. Therefore, the eye diagram after the PWL is still closed for the Vpp of $1.75 \mathrm{~V}$, with a slightly improved BER than using the FFE2. The eye diagram with the Vpp of $4.75 \mathrm{~V}$ after the FFE2 is extremely closed and skewed. By using the PWL, the BER improves a lot compared with utilizing the FFE2. It confirms that the PWL equalizer is very effective for the case of severe adiabatic chirp induced performance degradation. In addition, we observe that the best BERs using the FFE2 and PWL are achieved at the Vpps of $2.25 \mathrm{~V}$ and 3.25 $\mathrm{V}$ because the PWL equalizer can compensate nonlinear distortion introduced by the high Vpp.
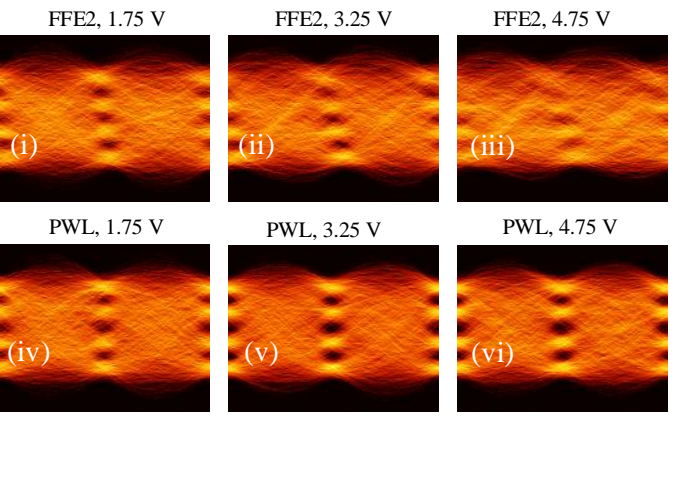

Fig. 5. BER curves versus RF input Vpps with FFE2 and PWL equalizers for $56 \mathrm{~Gb} / \mathrm{s}$ after 40km transmission, and $84 \mathrm{~Gb} / \mathrm{s}$ after $20 \mathrm{~km}$ transmission cases. Eye diagrams of 56 Gb/s PAM-4 after 40 km transmission with FFE2 (i-iii) and PWL (iv-vi) with RF input Vpps of 1.75 V, $3.25 \mathrm{~V}$, and $4.75 \mathrm{~V}$.

\section{Computational Complexity of the PWL and $2^{\text {nd }}$ Order Volterra Equalizer}

The PWL equalizer has been demonstrated as an effective equalizer to correct eye skew in the previous sections. On the other hand, Volterra equalizer is also a famous equalizer to compensate nonlinear distortions, including eye skew induced by DML adiabatic chirp and fiber dispersion. Therefore, we made comparisons of computational complexity (CC) between the PWL equalizer and the $2^{\text {nd }}$ order Volterra equalizer. Taking into account the high computation complexity of high order terms and $2^{\text {nd }}$ order Volterra equalizer can sufficiently equalize the eye skewed signal, up to $2^{\text {nd }}$ order Volterra equalizer is used for the comparison [8]. It can be implemented in the following form,

$$
y(k)=\sum_{l_{1}=0}^{L_{2}-1} \sum_{l_{2}=0}^{l_{1}} h_{2}\left(l_{1}, l_{2}\right) \prod_{m=1}^{2} x\left(k-l_{m}\right)+\sum_{l_{1}=1}^{L_{1}-1} h_{1}\left(l_{1}\right) x\left(k-l_{1}\right) .
$$

where $x\left(k-l_{m}\right)$ is the $\left(k-l_{m}\right)$ th sample of the received signal sequence $x, h_{1}\left(l_{1}\right)$ and $h_{2}\left(l_{1}, l_{2}\right)$ are the $1^{\text {st }}$ and $2^{\text {nd }}$ order
Volterra kernels, which defines the number of involved samples. Its computational complexity can be expressed by the number of multiplexers:

$$
C C_{\text {volterra }}=L_{1}+L_{2} *\left(L_{2}+1\right) .
$$

The implementation form and computational complexity of PWL equalizers are expressed in (2) and (5) in section II, respectively. Since the threshold set of $\{-\lambda, \lambda\}$ for PWL is adopted, 11-tap pre-equalizer and three parallel FFEs are used. Specifically, in (5) and (7), $L_{0}$ equals 11, $L_{1}, L_{2}$ and $L_{3}$ are the same, representing the memory length of each segment FFE equalizer of the PWL. To benchmark the computational complexity of the $2^{\text {nd }}$ order Volterra, we vary the number of taps of each linear equalizer in PWL to achieve the BER of $3.8 \times 10^{-3}$ at the ROP of $5.6 \mathrm{dBm}$, for the cases of both $56 \mathrm{~Gb} / \mathrm{s}$ over $40 \mathrm{~km}$ and $84 \mathrm{~Gb} / \mathrm{s}$ over $20 \mathrm{~km}$. The red and blue dotted lines in Figs. 6(a) and 6(b) are BER and computational complexity of $2^{\text {nd }}$ order Volterra with $L_{1}=35$ and $L_{2}=11$ for the $56 \mathrm{~Gb} / \mathrm{s}$ signal and $L_{1}=65$ and $L_{2}=15$ for the $84 \mathrm{~Gb} / \mathrm{s}$ 
signal, respectively. According to the calculated results, the PWL equalizer achieves a similar BER performance as a $2^{\text {nd }}$ order Volterra equalizer, yet with a $61.4 \%$ and $40.4 \%$ reduction in computational complexity for $56 \mathrm{~Gb} / \mathrm{s}$ over $40 \mathrm{~km}$ and 84 $\mathrm{Gb} / \mathrm{s}$ over $20 \mathrm{~km}$ transmissions, respectively.
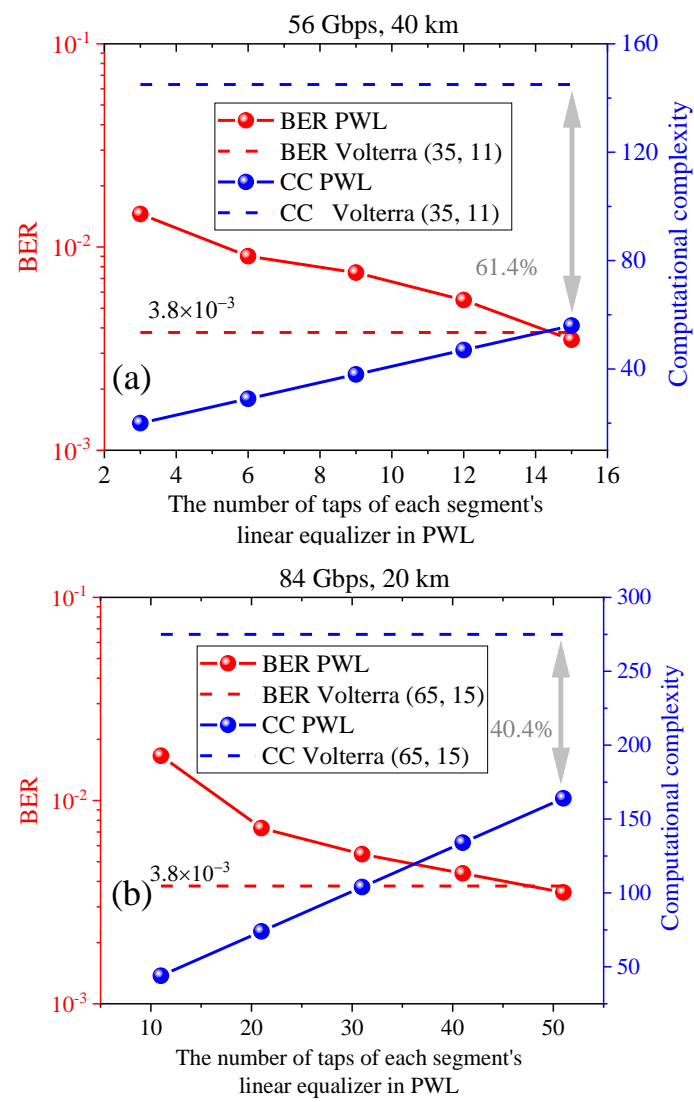

Fig. 6. BER and computational complexity comparison of PWL and Volterra when varying taps of each FFE equalizer of each segment in PWL for (a) 56 $\mathrm{Gb} / \mathrm{s}$ over $40 \mathrm{~km}$ and (b) $84 \mathrm{~Gb} / \mathrm{s}$ over $20 \mathrm{~km}$ transmissions.

\section{CONCLUSION}

We propose the piecewise linear equalizer, for the first time, by using the amplitude threshold decomposition to correct eye skew induced by the DML adiabatic chirp and fiber dispersion. The PWL equalizer consists of three parts: dividing each received sample into several segments according to the predefined amplitude threshold sets, parallel multiple linear equalizers, and final addition. By using this PWL equalizer, eye skew can be corrected effectively in $56 \mathrm{~Gb} / \mathrm{s}$ PAM-4 signal over $40 \mathrm{~km}$ transmission, and $84 \mathrm{~Gb} / \mathrm{s}$ PAM-4 signal over $20 \mathrm{~km}$ transmission. To optimize the performance and reduce the complexity of the PWL equalizer, we also analyse the impact of the number of pieces, segment positions by varying $\lambda$ of two threshold sets $\{-\lambda, 0, \lambda\}$ and $\{-\lambda, \lambda\}$ from 0 to 4 .

According to the experimental results, the PWL with the threshold sets of $\{-1,1\}$ and $\{-1.2,1.2\}$ achieves the best performance for $56 \mathrm{~Gb} / \mathrm{s}$ PAM-4 over $40 \mathrm{~km}$, and $84 \mathrm{~Gb} / \mathrm{s}$ PAM-4 over $20 \mathrm{~km}$ transmission with the BERs below the HD-FEC limit. In addition, we compared the computational complexity of the PWL with that of $2^{\text {nd }}$ order Volterra equalizer at the similar BER of $3.8 \times 10^{-3}$, and the results show the PWL can achieve $61.4 \%$ and $40.4 \%$ computational complexity reduction for $56 \mathrm{~Gb} / \mathrm{s}$ PAM-4 over $40 \mathrm{~km}$, and $84 \mathrm{~Gb} / \mathrm{s}$ PAM-4 over $20 \mathrm{~km}$ transmission, respectively. Finally, the PWL can be used not only in the DML-DD system to solve the eye skew but also in other nonlinear-amplitude-characteristic application scenarios, such as using the PWL for the patterning effect in semiconductor optical amplifier (SOA) and for compensating the chirp in vertical-cavity surface-emitting laser (VCSEL) and EML.

\section{ACKNOWLEDGMENT}

Authors acknowledge valuable discussion with Stenio Magalhaes Ranzini from DTU Fotonik, Technical University of Denmark.

\section{REFERENCES}

[1] Z. Li, L. Yi, H. Ji, and W. Hu, "100-Gb/s TWDM-PON based on 10G optical devices," Opt. Express, vol. 24, no. 12, pp. 12941-12948, 2016.

[2] N. Stojanovic, C. Prodaniuc, L. Zhang, J. Wei, S. Calabro, T. Rahman, and C. Xie, "Four-Dimensional PAM-7 trellis coded Modulation for Data Centers," IEEE Photonics Technol. Lett., vol. 31, no. 5, pp. 1-6, 2019.

[3] C. Kottke, C. Caspar, V. Jungnickel, R. Freund, M. Agustin, J. R. Kropp, and N. N. Ledentsov, "High-Speed DMT and VCSEL-Based MMF Transmission Using Pre-Distortion," J. Light. Technol., vol. 36, no. 2, pp. 168-174, 2017.

[4] K. Zhong, X. Zhou, T. Gui, L. Tao, Y. Gao, W. Chen, J. Man, L. Zeng, A. Lau, and C. Lu, "Experimental study of PAM-4, CAP-16, and DMT for $100 \mathrm{~Gb} / \mathrm{s}$ Short Reach Optical Transmission Systems," Opt. Express, vol. 23, no. 2, pp. 1176-1189, 2015.

[5] K. Zhang, H. He, H. Xin, W. Hu, S. Liang, D. Lu, and L. Zhao, "Chirp-aided power fading mitigation for upstream $100 \mathrm{~km}$ full-range long reach PON with DBR DML," Opt. Commun., vol. 407, pp. 63-68, 2018.

[6] S. H. Bae, H. Kim, and Y. C. Chung, "Transmission of 51.56-Gb/s OOK signal using $155-\mu \mathrm{m}$ directly modulated laser and duobinary electrical equalizer," Opt. Express, vol. 24, no. 20, pp. 22555-22562, 2016.

[7] R. Motaghiannezam, T. Pham, A. Chen, T. Du, C. Kocot, J. Xu, and B. Huebner, "52 Gb/s PAM4 receiver sensitivity study for 400GBase-LR8 system using directly modulated laser," Opt. Express, vol. 24, no. 7, pp. 7374-7380, 2016.

[8] M. Li, Y. Chen, Y. Liang, H. Zhang, E. Marentes, Q. Li, Y. Wang, and J. Zheng, "Demonstration of $100 \mathrm{~Gb} / \mathrm{s}$ per lambda PAM4 transmission with $1310 \mathrm{~nm}$ and $1330 \mathrm{~nm}$ directly modulated lasers," 2019 Opt. Fiber Commun. Conf. Exhib., p. W3A.6, 2019.

[9] W. Wang, H. Li, P. Zhao, Z. Zhang, D. Zang, C. Wang, L. Li, L. Ma, Y. Liu, N. Zhu, and Y. Lu, "Advanced digital signal processing for reach extension and performance enhancement of $112 \mathrm{~Gb} / \mathrm{s}$ and beyond direct detected DML-based transmission,” J. Light. Technol., vol. 37, no. 1, pp. 163-169, 2019.

[10] J. Zhang, J. Yu, X. Li, Y. Wei, K. Wang, L. Zhao, W. Zhao, M. Kong, X. Pan, B. Liu, and X. Xin, "100 Gbit/s VSB-PAM-n IM/DD transmission system based on $10 \mathrm{GHz}$ DML with optical filtering and joint nonlinear equalization," Opt. Express, vol. 27, no. 5, pp. 6098-6105, 2019.

[11] N. Diamantopoulos, H. Nishi, W. Kobayashi, and K. Takeda, "On the Complexity Reduction of the Second-Order Volterra nonlinear equalizer for IM/DD systems," J. Light. Technol., vol. 37, no. 4, pp. 1214-1224, 2019.

[12] A. G. Reza and J. K. K. Rhee, "Nonlinear equalizer based on neural networks for PAM-4 signal transmission using DML," IEEE Photonics Technol. Lett., vol. 30, no. 15, pp. 1416-1419, 2018.

[13] X. Miao, M. Bi, J. Yu, L. Li, and W. Hu, "SVM-Modified-FFE Enabled Chirp Management for 10G DML-based 50Gb/s/ $\mathrm{PAM}-4$ IM-DD PON," 2019 Opt. Fiber Commun. Conf. Exhib., p. M2B.5, 2019.

[14] L. Yi, T. Liao, L. Huang, L. Xue, P. Li, and W. Hu, "Machine Learning for $100 \mathrm{~Gb} / \mathrm{s} / \lambda$ Passive Optical Network," J. Light. Technol., vol. 37, no. 6, pp. 1621-1630, 2019.

[15] N. Kikuchi, R. Hirai, and T. Fukui, "Non-linearity Compensation of High-Speed PAM-4 Signals from Directly-Modulated Laser at High 
Extinction Ratio,” Eur. Conf. Opt. Commun. ECOC, p. M.2.B.4, 2016.

[16] N. Stojanović, Z. Qiang, C. Prodaniuc, and F. Karinou, "Eye Deskewing Algorithms for PAM Modulation Formats in IM-DD Transmission Systems," 2017 Opt. Fiber Commun. Conf. Exhib., p. Tu2D.4, 2017.

[17] K. Zhang, Q. Zhuge, H. Xin, M. Morsy-Osman, E. EI-Fiky, L. Yi, W. Hu, and D. V. Plant, "Intensity directed equalizer for the mitigation of DML chirp induced distortion in dispersion-unmanaged C-band PAM transmission," Opt. Express, vol. 25, no. 23, p. 28123-28135, 2017.

[18] K. Zhang, Q. Zhuge, H. Xin, M. Morsy-Osman, E. EI-Fiky, L. Yi, W. Hu, and D. V. Plant, "Intensity-directed Equalizer for Chirp Compensation Enabling DML-based 56Gb/s PAM-4 C-band Delivery over $35.9 \mathrm{~km}$ SSMF," Eur. Conf. Opt. Commun. ECOC, p. M.2.F.1, 2017.

[19] S. B. Gelfand, C. S. Ravishankar, and E. J. Delp, "Tree-Structured Piecewise Linear Adaptive Equalization," IEEE Trans. Commun., vol. 41, no. 1, pp. 70-82, 1993.

[20] E. A. Heredia and G. R. Arce, "Piecewise linear system modeling based on a continuous threshold decomposition," IEEE Trans. Signal Process., vol. 44, no. 6, pp. 1440-1453, Jun. 1996.

[21] N. Eiselt, H. Griesser, J. Wei, A. Dochhan, R. Hohenleitner, M. Ortsiefer, M. Eiselt, C. Neumeyr, J. Olmos and I. Monroy, "Experimental demonstration of $56 \mathrm{Gbit} / \mathrm{s}$ PAM- 4 over $15 \mathrm{~km}$ and $84 \mathrm{Gbit} / \mathrm{s}$ PAM- 4 over $1 \mathrm{~km}$ SSMF at $1525 \mathrm{~nm}$ using a $25 \mathrm{G}$ VCSEL," Eur. Conf. Opt. Commun. ECOC, vol. 2016-Septe, no. 1, p. Th.1.C.1, 2016.

[22] N. Eiselt, H. Griesser, J. Wei, R. Hohenleitner, A. Dochhan, M. Ortsiefer, M. Eiselt, C. Neumeyr, J. Olmos and I. Monroy, "Experimental demonstration of $84 \mathrm{~Gb} / \mathrm{s}$ PAM-4 over up to $1.6 \mathrm{~km}$ SSMF using a 20-GHz VCSEL at $1525 \mathrm{~nm}$," J. Light. Technol. vol. 35, no. 8, pp. 1342-1349, 2017.

[23] L. M. Zhang and F. R. Kschischang, "Staircase codes with $6 \%$ to $33 \%$ overhead," J. Light. Technol. vol. 32, no. 10, pp. 1999-2002, 2014. 